\section{CPS-139 THE PIRAMID STUDY: PATIENTS TREATED BY MYELOMA ORAL MEDICATION HAVE A BAD COMPLIANCE}

N Allaire*, JF Huon, P Nizet, C Dubreuil, V Burgeot, I Rouiller, L Flet, M Tching-Sin. CHU Nantes, Pharmacy, Nantes, France

\subsection{6/ejhpharm-2019-eahpconf.288}

Background Like other cancerous diseases, there are now many oral treatments for haematological malignancies. These oral products have a lot of advantages, but their effectiveness depends on patient compliance. Do they have a good effect?

Purpose This study aimed to assess patient medication compliance for myeloma oral treatments (Imids: Thalidomid, Lenalidomid, Pomalidomid) and to identify factors associated with medication compliance by adult outpatients.

Material and methods A cross-sectional prospective study was conducted at a university teaching hospital from January to August 2018.

Investigational medication compliance was assessed at the time of the renewal dispensation using the Morisky questionnaire. Other questionnaires were completed by outpatients at home: SF-36 quality of life, SATMED-Q satisfaction of treatment and HLQ health literacy. Demographic and other baseline data were collected using self-administered questionnaires.

Results Of the 62 participants, 36 (58.1\%) were male, and the median age was 69 years (range: $43-85$ years). The mean compliance Morisky score was 7.12/8\% and $45.9 \%$ of the patients had a score of $8 / 8$ (good observance). Patients were divided into two groups for the analysis: the good adherence one (score $>7, \mathrm{n}=28$ ) and the medium/poor adherence group (score $\leq 7, \mathrm{n}=33$ ).

In univariate analysis, the Morisky medication compliance score was negatively correlated with existence of a tip to remember drug intake $(\mathrm{p}=0.0234)$, being a member of a patients' association $(\mathrm{p}=0.0118)$ and positively correlated with treatment satisfaction $(\mathrm{p}=0.0081)$, perceived health $(p=0.0175)$, a good psychic health $(p=0.0119)$ and few limitations associated with it $(\mathrm{p}=0.0047)$.

Conclusion Only half the included outpatients had an optimal adherence of their medication. Having a poor satisfaction of their treatment and a poor psychic health were important risk factors for inadequate medication compliance.

This and other data suggest that hospitals and health professionals should encourage initiatives aimed at improving medication compliance in adults treated by Imids. Therapeutic education is not the easiest solution, due to the necessity of training. Pharmaceutic interviews could be easier to set up. To maximise the efficiency of these interventions, they should be aimed to poor compliance assumed patients.

\section{REFERENCES AND/OR ACKNOWLEDGEMENTS}

No conflict of interest.

\section{CPS-140 HOW PHARMACISTS CAN IMPROVE PATIENTS' CARE MANAGEMENT IN SUBCUTANEOUS ANTIPSORIATIC BIOLOGIC THERAPY}

${ }^{1} \mathrm{H}$ Benoist* ${ }^{*} \mathrm{~A}$ Dinahet, ${ }^{2} \mathrm{C}$ Clement, ${ }^{1} \mathrm{G}$ Saint-Lorant. ${ }^{1}$ Caen University Hospital, Pharmacy, Caen, France; ${ }^{2}$ Caen University Hospital, Dermatology, Caen, France

10.1136/ejhpharm-2019-eahpconf.289
Background Subcutaneous antipsoriatic biologic therapy (SCABT) facilitates ambulatory care. However, it requires a therapeutic education of patients (TEP) to enable patients to acquire the necessary skills for the proper use of medications and to ensure safe administration.

Purpose The aim of this study was to improve patients' care management on SCABT.

Material and methods This work took place in the dermatology unit (10 beds) of a university hospital. A patient and nurse survey was conducted during TEP sessions in order to identify requirements. TEP sessions took place directly after the dermatological consultation and were dedicated to one patient. A questionnaire evaluating nurses' knowledge on SCABT was distributed to each nurse $(n=10)$. Following the implementation of pharmacist-led actions, a second round of the questionnaire was distributed. A feedback session of the nurses and a patient satisfaction survey were also done.

Results The survey of six patients showed that 100\% did not read or still lose information supports from pharmaceutical companies. The nurses survey $(n=6)$ had highlighted their need for adapted tools. The first round of the nurses $(n=10)$ questionnaire showed less than $10 \%$ of correct answers regarding each SCABT characteristic. A nurse training course of 1 hour made by pharmacists was presented to nurses $(n=5)$. This presentation contained all the essential information on SCABT. Leaflets of all SCABT were carried out in a multi-disciplinary approach to remind patients of all key points of SCABT. A dosing regimen on the leaflets allowed the patient to trace their home injections which were checked by the nurse on the following appointment. A poster 'Guide SCABT' was elaborated in order to sum up all information. The results of the nurse post-training questionnaire $(n=4)$ was as follows: $100 \%$ of nurses knew methods of preservation, $100 \%$ dispensing modalities, $100 \%$ waste management and 50\% SCABT characteristics. The 2 month feedback showed a general satisfaction of all patients $(n=6)$ and all caregivers $(n=7)$.

Conclusion This multidisciplinary approach helps meet patients' expectations and creates a dynamic and thorough TEP approach. It confirms that clinical pharmacy services help answer patients' and caregivers' needs.

\section{REFERENCES AND/OR ACKNOWLEDGEMENTS}

No conflict of interest.

\section{CPS-141 ECONOMIC IMPACT OVER THE PAST 4 YEARS ASSOCIATED WITH BIOLOGICAL THERAPY OPTIMISATION IN RHEUMATIC DISEASES PATIENTS}

${ }^{1}$ I Cavada Carranza*, ${ }^{1} \mathrm{FJ}$ Alonso-Zazo, ${ }^{1} \mathrm{ME}$ Martínez-Núñez, ${ }^{1} \mathrm{R}$ Vázquez-Sánchez, 1J Sánchez-Rubio-Ferrández, ${ }^{2} A$ Aragón-Díez, ${ }^{2} \mathrm{JM}$ Rodríguez-Heredia, ${ }^{1} \mathrm{~T}$ Molina-García. ${ }^{1}$ Hospital Universitario de Getafe, Pharmacy Department, Madrid, Spain; ${ }^{2}$ Hospital Universitario de Getafe, Rheumatology Department, Madrid, Spain

\subsection{6/ejhpharm-2019-eahpconf.290}

Background Therapeutic decision-making for biologic-therapies (BT) dose optimisation should be based on optimal disease activity results. The goal of BT optimisation is to guarantee long-term effectiveness and safety, maximising economic savings.

Purpose To evaluate BT optimisation patterns in patients with rheumatic diseases (RD) and associated economic savings. Material and methods Observational and retrospective study. 
All patients with RD (rheumatoid arthritis (RA), spondyloarthropathies (SA) and psoriatic arthritis (PSA)) treated with BT from January 2014 to June 2018 were included.

BT optimisation, by dose reduction or prolonging the dosing interval, was indicated when patients had more than 6 months in clinical remission (DAS28 $<2.6$ for RA and PSA and BASDAI $<2$ for SA) or minimal clinical activity (DAS28 $<3.2$ and BASDAI <4).

Variables were described as frequencies and means. Diagnosis, subcutaneous BT (Abatacept, Adalimumab, Certolizumab, Etanercept, Golimumab, Secukinumab, Tocilizumab and Ustekinumab), dose regimens, total treatment duration, time on BT optimisation (TO) and treatment costs were collected.

Cost savings were calculated per patient by comparing optimisation treatment costs to conventional treatment and globally by comparing real cost to theoretical conventional doses cost.

Results A total of 448 patients were included in the study, receiving 579 BT treatments. Switching was observed in $29 \%$. From all patients, $47 \%$ were in BT optimisation (according to diagnosis: $53.7 \%$ with RA, followed by $47.7 \%$ with SA and $33.1 \%$ with PSA).

Sixty per cent of patients with BT optimisation were treated with adalimumab and etanercept, being also the most common BT used in RD treatment.

Mean TO duration was 2.2 years. The longest TO were achieved with adalimumab and golimumab (2.7 years) and PSA patients preserved BT optimisation for a mean of 2.8 years.

BT optimisation allowed a 50\% saving per patient against the use of conventional therapy resulting in a reduction of the total cost of $€ 3,000,000$ in the past 4 years, which represents a total economic savings of $21 \%$.

Conclusion Therapeutic decision-making based on validated disease activity scales has allowed BT optimisation in approximately $50 \%$ of patients with RD.

Patients remain clinically controlled after BT optimisation for a mean time of 2 years.

BT optimisation allows a reduction in costs while maintaining effectiveness.

\section{REFERENCES AND/OR ACKNOWLEDGEMENTS}

No conflict of interest.

\section{CPS-142 ADJUVANT CHEMOTHERAPY AND RELAPSE- ASSOCIATED PROGNOSTIC FACTORS IN OPERABLE BREAST CANCER}

${ }^{1,2} \mathrm{~B}$ François, ${ }^{3} \mathrm{~A}$ Benider, ${ }^{4} \mathrm{~S}$ Housbane, ${ }^{1} \mathrm{H}$ Chorouk ${ }^{*},{ }^{1,2} \mathrm{Z}$ Lhoussaine, ${ }^{1,2} \mathrm{~S}$ Derfoufi. ${ }^{1} / B N$ Rochd University Hospital Centre, Pharmacy Service, Casablanca, Morocco; ${ }^{2}$ Medical and Pharmaceutical College - University Hassan II, Laboratory of Drug Sciences- Biomedical Research and Biotechnology, Casablanca, Morocco; ${ }^{3}$ IBN Rochd University Hospital Centre, Mohammed VI Centre for the Treatment of Cancers, Casablanca, Morocco; ${ }^{4}$ Medical and Pharmaceutical College-University Hassan II, Laboratory of Medical Informatics, Casablanca, Morocco

\subsection{6/ejhpharm-2019-eahpconf.291}

Background Breast cancer is characterised by its extreme frequency. Its management is now dependent on the prognostic factors according to the guidelines of the experts.

Purpose The aim of our study was to analyse the adjuvant systemic management of operable breast cancer in Morocco, relapsefree survival and the recurrence-associated prognostic factors.

Material and methods This was a retrospective study of patients treated for breast cancer at the Mohamed VI Centre for Cancer Treatment of Casablanca for 3 years, from 2010 to 2012. Data related to management strategies, relapse and prognostic factors were retrospectively collected from patients' records in 2018 and statistical analyses were performed using the SPSS 20.0 software. Relapse-free survival was calculated with the Kaplan-Meier method, and compared with the Logrank test with an alfa risk of 5\%. Univariate and multivariate logistic regression were used to identify recurrence-associated factors.

Results Six-hundred and one patients including six males were included in our study. The mean age at diagnosis was $49.2 \pm 10.8$ years. The majority of tumours were ductal carcinomas of 2 to $5 \mathrm{~cm}$ and grade II, with luminal/HER2 negative phenotype, stage II and III. Ninety-three per cent of patients had an average of six cycles of chemotherapy, mainly the AC60-T and FEC100-T protocols. Tamoxifen was prescribed to $87 \%$ of patients with luminal tumours and the HER2-directed therapy was prescribed to $23 \%$ of patients. The 5 year relapse-free survival was $77.5 \%$ and the hormonotherapy significantly improved it, while HER2 targeting therapy showed no significant effect on relapse-free survival. The recurrence-associated factors were tumour size, grade SBR, the presence of vascular emboli and the involvement of axillary lymph nodes.

Conclusion Our results show that systemic management and relapse-free survival depend on tumour phenotype, and highlight prognostic factors known to be associated with relapse.

\section{REFERENCES AND/OR ACKNOWLEDGEMENTS}

We are thankfull to Professor Samir Ahid, member of the Pharmacoeconomics and Pharmacoepidemiology Research team at the Faculty of Medicine and Pharmacy of Rabat, Morocco for his help and support.

No conflict of interest.

\section{CPS-143 EVALUATION OF KNOWLEDGE OF PATIENTS WITH INFLAMMATORY BOWEL DISEASE TREATED BY THE REFERENCE PRODUCT CONCERNING BIOSIMILARS: ROLE OF CLINICAL PHARMACISTS}

${ }^{1}$ E Coget ${ }^{*},{ }^{1} P$ Laffont-Lozes, ${ }^{1} V$ Velasco Gonzalvo, ${ }^{1} D$ Huc, ${ }^{2} G$ Pineton de Chambrun, ${ }^{2} \mathrm{R}$ Altwegg, ${ }^{2} \mathrm{P}$ Blanc, ${ }^{2} \mathrm{GP}$ Pageaux, ${ }^{1} \mathrm{D}$ Rosant, ${ }^{1} \mathrm{C}$ Breuker. ${ }^{1} \mathrm{CHU}$ Saint-Eloi, Pharmacy, Montpellier, France; ${ }^{2} \mathrm{CHU}$ Saint-Eloi, Gastroenterology, Montpellier, France

\subsection{6/ejhpharm-2019-eahpconf.292}

Background Anti-TNF monoclonal antibodies such as infliximab have revolutionised the treatment of patients with inflammatory bowel disease (IBD). For a few years several biosimilars of the reference product (RP) have reached the European market and switching IBD patients from original infliximab to biosimilars is the subject of debate.

Purpose The aim of our study was to investigate IBD patients' knowledge about infliximab biosimilars and their judgement concerning switching to a biosimilar.

Material and methods We conducted a prospective observational study over a 5 month period (May to September 2018) in the outpatient clinic of the Gastroenterology Department at a tertiary referral centre. Clinical pharmacists performed a standardised interview of consecutive adult IBD patients treated with RP. The interview included general information about anti-TNF monoclonal antibodies and biosimilars, and a questionnaire on patients' acceptance of switching to a biosimilar. For each patient included, a pharmaceutical note was edited 